\title{
How to Move a Ventilated Baby From Bed/Incubator to Skin-to Skin In a Safe Way
}

Hoeeg $J$

Neonanalklinikken, Rigshospitatet

Copenhagen, Denmark

\section{Aims}

For premature and full term infants who need help from a ventilator for breathing, it can be difficult to hold the infant skinto-skin and often depends on the nurses who are present at the time. Lying skin-to-skin is a way to treat premature babies. The specific aims of this project were:

1. To increase the opportunity for skin-to-skin contact for as many infants as possible, as early as possible, including those intubated.

2. To develop a formal guideline: How to move the baby from bedlincubator skin-to-skin with parents in a safe way to help the families, nurses and physicians.

\section{Methods}

The literature recommends disconnecting the intubated infant when moving the infant. The project set out to show that moving an infant to their parent for skin-to-skin while still connected to the ventilator is possible. A literature review was done, and based on that, plus many in-depth discussions with NICU staff, a set of procedures and guidelines were developed. The steps and procedures were documented with photographs showing how simply and beautifully one can move an infant from bed/incubator to their parent. The guideline: How to move the baby from bed/incubator skin-to-skin with parents in a safe way was completed in two years, and the nurses and doctors work together to use it and make skin-to-skin a reliable part of the infant's treatment process.

\section{Conclusion}

Skin-to-skin care is an important component of NIDCAP care recommendations and a way to strengthen the family centered care. With the development of the guidelines on how to safely move intubated infants into skin-to-skin positioning, the unit now has the opportunity to offer skin-to-skin holding to all children in the unit. 